\title{
Vitiligo, P.N.E.I. and Low Dose Cytokines and Growth Factors
}

\section{Torello lotti ${ }^{1}$ and Alessandro Perra ${ }^{2 *}$}

${ }^{1}$ Professor \& Chair of Dermatology, University of Rome "G.Marconi", Italy

${ }^{2}$ President, GUNA Foundations, Italy

Vitiligo is a skin disorder characterized by a progressive depigmentation which is caused by the loss of melanocytes at the cutaneous level. The causes of melanocyte loss are still unclear, but a relevant number of observations lead researchers to ascribe cellular immunity as having an important role in vitiligo pathogenesis. In vitiligo, the observed imbalance in cytokine expression at cutaneous lesions level both on the border of affected area with healthy skin and in the center of the spot, is probably due to a shift of the immune system with a prevalence of Th1/Th17 (high IL-1 and IL-17 levels) response instead of a Tregs/Th2 one (low IL-4 level) and may be part of etiology of this autoimmune disease [1-3]. TNF- $\alpha$ also has a pivotal role in oxidative stress-mediated cytotoxicity directed against melanocytes and keratinocytes and sustained by $\mathrm{T}$-cells and B-cells activation $[4,5]$.

Apoptosis of keratinocytes is a key event in Vitiligo onset. Keratinocytes produce a number of growth factors that support the viability of the melanocytes through paracrine action [6]. Among these factors, the basic fibroblast growth factor (bFGF or FGF2) is a potent regulator of melanocytes proliferation, differentiation and function; there is an inverse correlation between the levels of TNF- $\alpha$ and bFGF in the presence of vitiligo and low levels of bFGF are responsible of melanocytes death and, finally, of skin lesion depigmentation [7]. TNF- $\alpha$ is, therefore, a key player of cell-mediated autoimmune response and inflammation, responsible of the keratinocytes apoptosis [8].

It is therefore arguable that the inflammatory phenomenon supported by this cytokine and the breaking down of the paracrine crosstalk between keratinocytes and melanocytes are central events in inducing and sustaining vitiligo and thus represents one of the key targets within a therapeutic strategy based on deep knowledge of the etiology of the disease.

Low-Dose Medicine starts from the innovative therapeutic concept of restoring physiology through activated low dose communicating (messenger-signaling) molecules such as hormones, neuropeptides, interleukins, and growth factors. The availability of low-dose medicines containing highly diluted and Sequentially Kinetic-Activated (SKA) cytokines and growth factors represents a unique opportunity for the study of an innovative immunological therapeutic approach for vitiligo treatment.

The therapeutic use of SKA low dose bFGF (Guna S.p.a. - Italy) is aimed at improving the stimulation of the residual population of melanocytes (via up-regulation of trans-membrane receptors) mimicking the paracrine signaling exerted, in physiological conditions, by keratinocytes. The effect of bFGF is improved if in association with the pool of anti-inflammatory cytokines that acts reducing the apoptotic phenomena and the cytotoxicity mediated by TNF- $\alpha$.

The anti-inflammatory SKS low dose molecules pool is composed by Anti-IL-1; IL-4; IL-10 and $\beta$-endorphin.

Anti-IL-1 is useful in the reduction of IL-1-mediated acute inflammation, present in perilesional region; IL-4 exerts an effective control against autoimmune triggers, reducing cell-mediated cytotoxicity; IL-10 is the opposing cytokine of both IL-1 and TNF-a and is effective in chronic inflammation control; $\beta$-endorphin, as $\beta$-endorphin oxidation reduces melanin synthesis (high level of oxydative stress is typical in vitiligo) [9].

The use SKA low dose bFGF and specific SKA low dose antiinflammatory cytokines pool represents a new therapeutic approach for vitiligo strictly based on disease etiology and aimed both to stop the disease progression, throughopposing chronic inflammation, and to induce skin repigmentation by direct stimulation of melanocytes.

\section{References}

1. Wang $C Q$, Cruz-Inigo AE, Fuentes-Duculan J, Moussai D, Gulati N, et al. (2011) Th17 cells and activated dendritic cells are increased in vitiligo lesions. PLoS One 6: e18907.

2. Sandoval-Cruz M, García-Carrasco $M$, Sánchez-Porras $R$, Mendoza-Pinto C, Jiménez-Hernández $M$, et al. (2011) Immunopathogenesis of vitiligo. Autoimmun Rev 10: 762-765.

3. Dwivedi M, Laddha NC, Arora P, Marfatia YS, Begum R (2013) Decreased regulatory T-cells and CD4(+)/CD8(+) ratio correlate with disease onset and progression in patients with generalized vitiligo. Pigment Cell Melanoma Res 26: 586-591.

4. Laddha NC, Dwivedi M, Mansuri MS, Gani AR, Ansarullah M, et al. (2013) Vitiligo: interplay between oxidative stress and immune system. Exp Dermatol 22: $245-250$.

5. Zailaie MZ (2005) Aspirin reduces serum anti-melanocyte antibodies and soluble interleukin-2 receptors in vitiligo patients. Saudi Med J 26: 1085-1091.

6. Lee AY (2012) Role of keratinocytes in the development of vitiligo. Ann Dermatol 24: 115-125.

7. Moretti S, Spallanzani A, Amato L, Hautmann G, Gallerani I, et al. (2002) New insights into the pathogenesis of vitiligo: imbalance of epidermal cytokines at sites of lesions. Pigment Cell Res 15: 87-92.

8. Zailaie MZ (2005) Aspirin reduces serum anti-melanocyte antibodies and soluble interleukin-2 receptors in vitiligo patients. Saudi Med J 26: 1085-1091.

9. Spencer JD, Gibbons NC, Rokos H, Peters EM, Wood JM, et al. (2000) Oxidative stress via hydrogen peroxide affects proopiomelanocortin peptides directly in the epidermis of patients with vitiligo. J Invest Dermatol 127:411-420.
*Corresponding author: Alessandro Perra, President, GUNA Foundations, Italy, Tel: 06891420157; E-mail: a.pizzoccaro@guna.it

Received September 25, 2014; Accepted September 27, 2014; Published September 30, 2014

Citation: lotti T, Perra A (2014) Vitiligo, P.N.E.I. and Low Dose Cytokines and Growth Factors. Pigmentary Disorders 1:e102. doi:10.4172/JPD.1000e102

Copyright: (c) 2014 lotti T, et al. the terms of the Creative Commons Attribution License, which permits unrestricted use, distribution, and reproduction in any medium, provided the original author and source are credited. 